\title{
Stage one adenocarcinoma of uterine cervix in a patient with systemic lupus erythematosus - a clinical case
}

\author{
Latchezar Tantchev ${ }^{1}$, Angel Yordanov², Veselin Marinov³, Andrey Kotzev ${ }^{4}$ \\ ${ }^{1}$ Obstetrics and Gynaecology Clinic, Acibadem City Clinic Hospital “Tokuda”, Sofia, Bulgaria \\ 2Department of Gynaecological Oncology, Medical University of Pleven, Bulgaria \\ ${ }^{3}$ General Surgery Clinic, Acibadem City Clinic Hospital “Tokuda”, Sofia, Bulgaria \\ ${ }^{4}$ Clinic of Gastroenterology, University Hospital for Active Treatment „Alexandrovska”, Sofia, Bulgaria
}

\begin{abstract}
Introduction: The systemic lupus erythematosus (SLE) is a comparatively rare chronic autoimmune disease in women of reproductive age, the pathogenesis and therapy of which also conditions the increased risk of development of malignant diseases.

Case report: We present a clinical case of a 38-year-old female patient with lupus and stage 1B1 adenocarcinoma of uterine cervix, in which robotic radical hysterectomy with pelvic lymph node dissection has been successfully performed. The strict primary and secondary prophylaxis for pathological changes of uterine cervix are of essential significance for patients with lupus.

Conclusions: SLE is a chronic autoimmune disease, which is frequently treated with immunosuppressors, and affects most often women in reproductive age. The combination of these conditions is associated with higher risk of infecting with HPV and occurrence of pre-cancer and cancer of uterine cervix. Based on these reasons women with SLE must undergo strict screening follow-up and vaccination against high-risk strains of HPV. In the cases of cancer of uterine cervix diagnosed in initial stage, the striving towards minimal traumatism and better quality of life of patient - and, at the same time, towards achievement of optimal radicality - directs to the choice of minimally invasive operative method such as the robotic surgery.
\end{abstract}

Key words: systemic lupus erythematosus, adenocarcinoma of uterine cervix, robotic surgery.

\section{Introduction}

The systemic lupus erythematosus (SLE) is a chronic inflammatory autoimmune disease of connective tissue, which affects mainly women in reproductive age [1]. The etiology has not been clarified, but it is regarded that the genetic predisposition is of leading significance, in combination with factors of environment, viral infections, and medications [2].

The standardized prevalence for the Republic of Bulgaria is 8.9 per 100,000 persons. Most frequently the beginning of disease is in the interval between sexual maturity and age of 40 years. The individuals, who become ill within this period, also have the most serious clinical manifestation of the disease [3].

The autoimmune genesis of disease and immunosuppressive treatment - which is a part of the therapeutic regimen - are associated with increase of the risk of occurrence of oncological diseases among patients with SLE [4].

\section{Case report}

This is a 38-year-old woman - with no history of pregnancies - with a cutaneous form of SLE diagnosed 12 years ago. Five of the criteria of the American Rheumatism Association from the year 1992 are present: butterfly rash; discoid lupus lesions; arthritis/arthralgias; immunological abnormalities (ANA, ADNA). Oral therapy with methylprednisolone and hydroxychloroquine has been administered for 12 years. SLE has been in remission - according to the clinical and laboratory results - for the last 2 years, and patient has been on maintenance therapy with reduced doses of the medical treatment. PAP smear 3A, and visible from colposcopy gross leukoplakia area (L2) were found on a prophylactic examination of patient; the leukoplakia area was with a section of mosaic (M2), and sizes of 1.5 $\times 2 \mathrm{~cm}$ in the region of exocervix, extending into the cervical canal. A loop excision of the described section was performed with histological result of adenocarcino- 


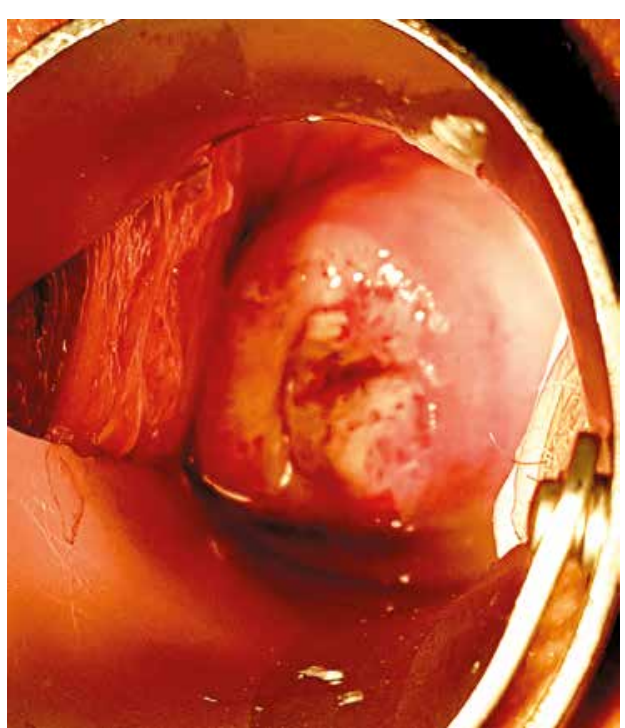

Fig. 1. Status of vaginal portion of uterine cervix after performing of large loop excision of transformation zone (LLETZ)

ma of uterine cervix and involvement of the resection lines. A repeated examination of histological preparation was accomplished, which confirmed the initial result - villoglandular adenocarcinoma with infiltration to the native squamous epithelium of uterine cervix. Patient was admitted at this Clinic for continuation of treatment (Fig. 1).

In view of the preceding disease (SLE) and histological result, a decision was made - after a thorough discussion - for performing of robotic radical hysterectomy with bilateral adnexectomy, combined with pelvic lymph node dissection (DaVinci $\mathrm{Si}^{\circledast}$, Intuitive Surgical). Based on the assessment of the attending rheumatologist, a preoperative preparation with Methylprednisolone $60+60+40+40+20 \mathrm{mg}$ was appointed, which began two days before the operation. Magnetic resonance imaging (MRI) of lesser pelvis, and pelvic and paraaortic lymph node chains, was performed, in which
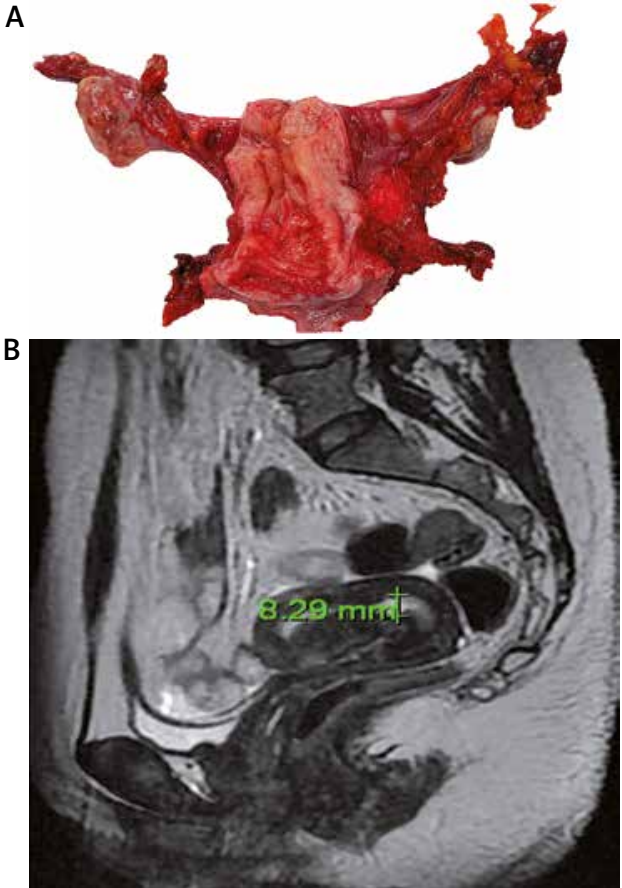

Fig. 2. A stable preparation after performing of robotic radical hysterectomy, and preoperative MRI examination of female reproductive organs

changes in uterine cervix were found with diameter of less than $10 \mathrm{~mm}$ (Fig. 2) and lymph nodes enlarged within referent range of up to $10 \mathrm{~mm}$ that were not suspected to be metastatic (Fig. 3). The operative intervention passed with no complications, with a total surgery time of 185 minutes, console time of 160 minutes, and total blood loss of $50 \mathrm{ml}$. The histological examination showed a high grade of glandular endocervical dysplasia (HCGIN), with no presence of residual carcinoma after the conization performed (Fig. 2).

Seventeen lymph nodes were removed on pelvic lymph node dissection - two of them were sent for fro-
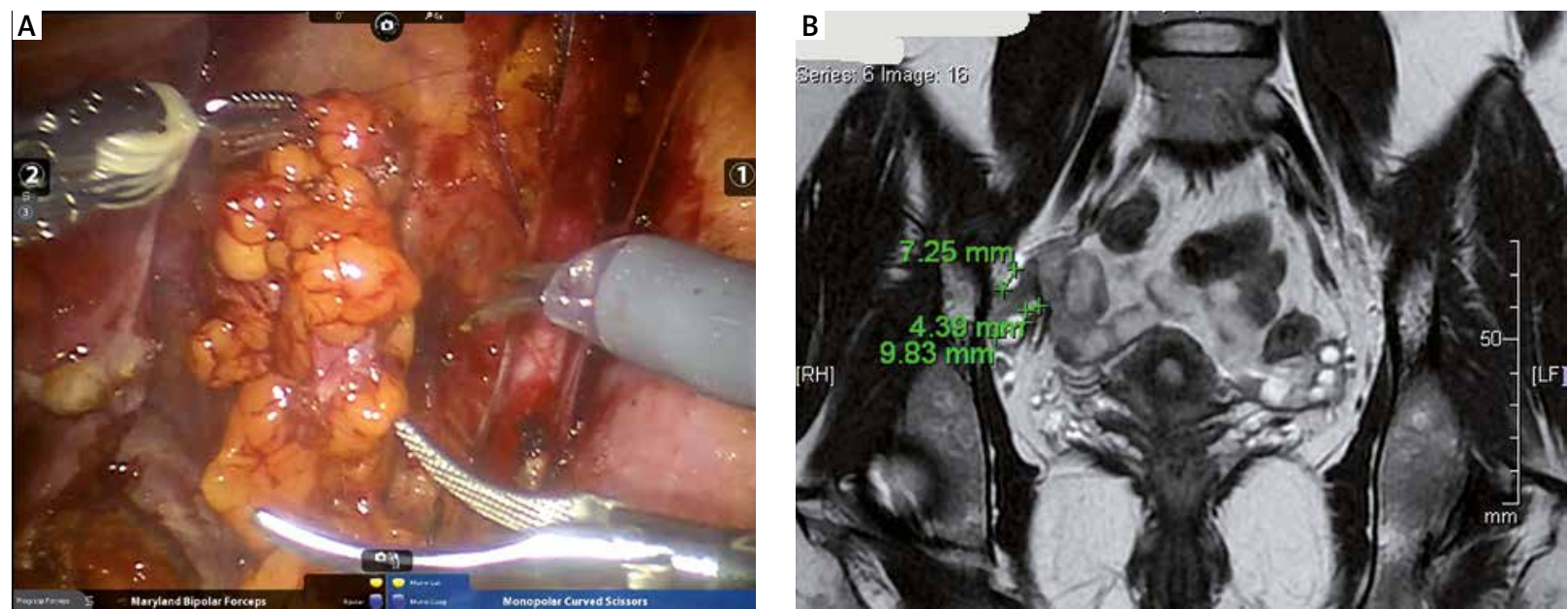

Fig. 3 Enlarged lymph nodes sent for frozen section analysis; MRI visualization of the latter lymph nodes 
zen section analysis due to their enlarged sizes, with no metastases found in them (Fig. 3). The final pathological examination did not reveal any lymphatic metastases.

The early postoperative period passed with significant lymphorrhea - respectively, $300 \mathrm{ml}, 500 \mathrm{ml}$, $600 \mathrm{ml}, 500 \mathrm{ml}, 600 \mathrm{ml}, 700 \mathrm{ml}, 700 \mathrm{ml}$ from the first to seventh day - which required a more prolonged hospitalization. Patient was discharged on seventh postoperative day, after which the abdominal drain was removed, and therapy was appointed for improvement of the condition of lymphedema. Within one month the lymphorrhea was significantly limited. In view of the histological result and according to the decision of the Oncological Committee, radiotherapy was not appointed. In the course of follow-up, presence of lymphocele was found three months after the intervention, in the area of left and right external iliac lymph node chains, with sizes of respectively $3 \times 2 \mathrm{~cm}$ and $2 \times 2 \mathrm{~cm}$; there was reduction of their sizes, with one centimeter each, on the sixth month, as a result of only medicinal treatment. Their drainage was not required due to the absence of symptoms. On the last follow-up examination, 9 months after the operation, patient felt well, with no data of persistence of disease.

\section{Discussion}

The treatment of cancer of uterine cervix according to the present-day understandings is predominantly surgical [2]. Due to the clinically determined stage 1B1 (a lesion of less than $2 \mathrm{~cm}$ upon performing of LLETZ), as well as the explicit wish of patient for minimally invasive procedure, we performed a robotic radical hysterectomy with pelvic lymph node dissection. The histological examination of the stable preparation, resection lines of vagina and parametrial tissue, as well as the number of removed lymph nodes (LN yield), showed surgical intervention of optimal radicality. The significant postoperative lymphorrhea could be due to both the operative technique and extensive pelvic lymph node dissection, and owing to manifestations on the part of reticuloendothelial system. This system reacts even in the beginning of SLE, because it is functionally burdened, especially when big amounts of immune complexes have to be eliminated, despite the fact that there are no laboratory and clinical data for activity of the disease at the moment of performing of the operative intervention. There are studies concerning the complications as inflamated lymphocyst after lymphadenectomy in gynecological malignancy $[5,6]$. The current trend is toward a sentinel lymph node biopsy $(\mathrm{SLN})$ rather than extensive systemic lymphadenectomy in early stages gynecological cancers according to some other authors [7, 8]. Data in the literature concerning the postoperative lymphedema in patients with SLE - after performed lymph node dissection - were not found.
A series of studies consider SLE as a predisposing factor for occurrence of oncological diseases. According to recent meta-analyses, SLE is associated with an increased risk for development of premalignant and malignant diseases of the uterine cervix as well $[4,9,10]$. Some authors considered that SLE could be a reason for non-effective removal of the infected with HPV epithelial cells [11]. Klumb et al. considered that patients subjected to long-term immunosuppression were more likely to develop pre-cancer lesions of uterine cervix, due to which they recommended a strict prophylactic program [12]. A national Swedish study, which included 4,976 women (23,136 person-years), who had SLE, reported of a high risk for cervical dysplasia, but no increased frequency of cancer of uterine cervix. The strict national screening program in connection with cancer of uterine cervix for secondary prophylaxis of the disease, has most probably some significance associated with that. The risk in the cohort treated with Azathioprine, Methotrexate, Ciclosporin, Rituximab, and Mycophenolate Mofetil is higher than the one in patients who took only antimalarial medications [13]. Other studies also confirmed the increased frequency of high-risk cervical lesions upon administration of immunosuppressive therapy $[14,15]$. Our patient also underwent long-term immunosuppressive therapy with prednisolones and Hydroxychloroquine (for more than four years), but not with the preparations mentioned above. On the other hand, there are studies, which did not show statistically significant dependence between the atypism of uterine cervix and preceding administration of immunosuppressors for therapy of SLE [16]. The data regarding the result of antimalarial medications, concerning the HPV infection, remain controversial $[17,18]$. The co-stimulatory molecules OX4OL [19] and CTLA4 [20] - according to studies concerning them probably play a key role in the pathogenesis of SLE and carcinogenesis, however further investigation is necessary for clarification of their pathogenetic significance.

The evidence for higher risk of persistence of HPV infection and occurrence of pre-cancer lesions, which on insufficiently good secondary prophylaxis - result in cancer of uterine cervix, reasonably pose the question for immunization against the most frequent strains of HPV in women with SLE. The opinion of collectives of survey reports varies. Soybilgic et al. and Mok et al., found lower seroconversion rates after vaccination against HPV [21, 22]. That is the reason why Santana et al. considered that the vaccination could not be accepted for effective prophylactic measure in patients with SLE, and shortening of the oncological prophylactic intervals is necessary [16]. According to us, the primary and secondary prophylaxis of cancer of uterine cervix is of particular significance especially in the risk groups as the one of patients with SLE. Vaccination in the described clinical case was not carried out, while the col- 
poscopical and cytological changes reported one year ago were not subject to further investigation, which resulted in delay of making of diagnosis.

\section{Conclusions}

SLE is a chronic autoimmune disease, which is frequently treated with immunosuppressors, and affects most often women in reproductive age. The combination of these conditions is associated with higher risk of infecting with HPV and occurrence of pre-cancer and cancer of uterine cervix. Based on these reasons women with SLE must undergo strict screening follow-up and vaccination against high-risk strains of HPV. In the cases of cancer of uterine cervix diagnosed in initial stage, the striving towards minimal traumatism and better quality of life of patient - and, at the same time, towards achievement of optimal radicality - directs to the choice of minimally invasive operative method such as the robotic surgery.

\section{Disclosure}

The authors report no conflict of interest.

\section{References}

1. Grein IH, Groot N, Lacerda MI, et al. HPV Infection and Vaccination in Systemic Lupus Erythematosus Patients: What We Really Should Know. Pediatr Rheumatol Online J 2016; 14: 12

2. Manolova I, Ivanova M, Aleksadrova E, et al. Association of trans-forming growth factor promoter polymorphism with systemic lupuserythematosus. Revmatologia (Sofia) 2012; 20: 52.

3. Miteva-Katrandzhieva TS, Stoilov R, Rhashkov R, et al. Epidemiology of Systemic Lupus Erythematosus in Bulgaria. Rare Dis Orphan Drugs 2018; 9: 18-23.

4. Song L, Wang Y, Zhang J, et al. The Risks of Cancer Development in Systemic Lupus Erythematosus (SLE) Patients: a Systematic Review and Meta-Analysis. Arthritis Res Ther 2018; 20: 270.

5. Ma X, Wang Y, Fan A, et al. Risk factors, microbiology and management of infected lymphocyst after lymphadenectomy for gynecologic malignancies. Arch Gynecol Obstet 2018; 298: 1195-1203.

6. Abdelazim IA, Zhurabekova G, Shikanova S, Karimova B. Risk factors, microbiology, and management of infected lymphocyst after lymphadenectomy for gynecologic malignancies: letter to the editor. Arch Gynecol Obstet 2019; 299: 1747-1748.

7. Abdelazim IA, Abu-Faza M, Zhurabekova G, et al. Sentinel lymph nodes in endometrial cancer update 2018. Gynecol Minim Invasive Ther 2019; 8: $94-100$.

8. Comprehensive Cervical Cancer Control: A Guide to Essential Practice, $2^{\text {nd }}$ ed. Annex 10, Cervical Cancer Treatment by FIGO Stage. World Health Organization, Geneva 2014. Available from: https://www.ncbi. nlm.nih.gov/books/NBK269617/

9. Cibere J, Sibley J, Haga M. Systemic Lupus Erythematosus and the Risk of Malignancy. Lupus 2001; 10: 394-400.

10. Zard E, Arnaud L, Mathian A, et al. Increased Risk of High Grade Cervical Squamous Intraepithelial Lesions in Systemic Lupus Erythematosus: A Meta-Analysis of the Literature. Autoimmun Rev 2014; 13: 730-735.

11. Dhar JP, Kmak D, Bhan R, et al. Abnormal cervicovaginal cytology in women with lupus: a retrospective cohort study. Gynecol Oncol 2001; 82: 4-6.
12. Klumb EM, Pinto AC, Jesus GR, et al. Are women with lupus at higher risk of HPV infection? Lupus 2010; 19: 1485-1491.

13. Wadstrom H, Arkema EV, Sjowall C, et al. Cervical Neoplasia in Systemic Lupus Erythematosus: a Nation Wide Study. Rheumatology(Oxford) 2017; 56: 613-619.

14. Feldman CH, Liu J, Feldman S, et al. Risk of High-Grade Cervical Dysplasia and Cervical Cancer in Women with Systemic Lupus Erythematosus Receiving Immunosuppressive Drugs. Lupus 2017; 26: 682-689.

15. Kim SC, Glynn RJ, Giovannucci E, et al. Risk of High-Grade Cervical Dysplasia and Cervical Cancer in Women with Systemic Inflammatory Diseases: a Population-Based Cohort Study.) Ann Rheum Dis 2015; 74: 1360-1367.

16. Santana IU, Gomes Ado N, Lyrio LD, et al. Systemic Lupus Erythematosus, Human Papilloma Virus Infection, Cervical Pre-Malignant and Malignant Lesions: a Systematic Review. Clin Rheumatol 2011; 30: 665672.

17. Ruiz-Irastorza G, Ugarte A, Egurbide MV, et al. Antimalarials May Influence the Risk of Malignancy in Systemic Lupus Erythematosus. Ann Rheum Dis Jun 2007; 66: 815-817.

18. Yu SL, Chan PK, Wong CK, et al. Antagonist-Mediated Down Regulation of Toll-Like Receptors Increases the Prevalence of Human Papilloma Virus Infection in Systemic Lupus Erythematosus. Arthritis Res Ther 2012; 14: R80.

19. Wang L, Li D, Fu Z, et al. Association of CTLA-4 Gene Polymorphisms with Sporadic Breast Cancer in Chinese Han Population. BMC Cancer 2007; 7: 1-7.

20. Ghaderi A. CTLA4 Gene Variants in Autoimmunity and Cancer: a Comparative Review. Iranian J Immunol 2011; 8: 127.

21. Soybilgic A, Onel KB, Utset $T$, et al. Safety and immunogenicity of the quadrivalent HPV vaccine in female Systemic Lupus Erythematosus patients aged 12 to 26 years. Pediatr Rheumatol Online J 2013; 11: 29.

22. Mok CC, Ho LY, Fong LS, To CH. Immunogenicity and safety of a quadrivalent human papillomavirus vaccine in patients with systemic lupus erythematosus: a case-control study. Ann Rheum Dis 2013; 72: 659664. 